\title{
The DPSK Signal Noncoherent Demodulation Receiver Based on the Duffing Oscillators Array
}

\author{
Yongqing $\mathrm{Fu}^{*, \ddagger}$, Yanan $\mathrm{Li}^{*, \S}$, Lin Zhang ${ }^{\dagger, \uparrow}$ and Xingyuan $\mathrm{Li}^{*}, \|$ \\ ${ }^{*}$ College of Information and Communication Engineering, \\ Harbin Engineering University, Harbin, \\ Heilongjiang 150001, P. R. China \\ ${ }^{\dagger}$ College of Underwater Acoustic Engineering, \\ Harbin Engineering University, Harbin, \\ Heilongjiang 150001, P. R. China \\ łfuyongqing@hrbeu.edu.cn \\ \$liyanan@hrbeu.edu.cn \\ 『zhanglin@hrbeu.edu.cn \\ "lixingyuan@hrbeu.edu.cn
}

Received December 9, 2015; Revised July 19, 2016

\begin{abstract}
Chaotic communication requires the knowledge of corresponding phase relationship between the primary phase of Duffing oscillator's internal driving force and the primary phase of the undetected signal. Currently, there is no method of noncoherent demodulation for DPSK (Differential Phase Shift Keying) signal and mobile communication signal by Duffing oscillator. To solve this problem, this study presents a noncoherent demodulation method based on the Duffing oscillators array and Duffing oscillator optimization. We first present the model of Duffing oscillator and its sensitivity to undetected signal primary phase. Then the zone partition is proposed to identify the Duffing oscillator's phase trajectory, and subsequently, the mathematical model and implementation method of the Duffing oscillators array are outlined. Thirdly, the Duffing oscillator optimization and its adaptive strobe technique are proposed, also their application to DPSK signal noncoherent demodulation are discussed. Finally, the design of new concept DPSK chaotic digital receiver based on the Duffing oscillators array is presented, together with its simulation results obtained by using SystemView simulation platform. The simulation results suggest that the new concept receiver based on the Duffing oscillator optimization of Duffing oscillators array owns better SNR (signal-to-noise ratio) threshold property than typical existing receivers (chaotic or nonchaotic) in the AWGN (additive white Gaussian noise) channel and multipath Rayleigh fading channel. In addition, the new concept receiver may detect mobile communication signal.
\end{abstract}

Keywords: Chaos; digital communication; nonlinear oscillator; receiver; signal detection.

This is an Open Access article published by World Scientific Publishing Company. It is distributed under the terms of the Creative Commons Attribution 4.0 (CC-BY) License. Further distribution of this work is permitted, provided the original work is properly cited. 


\section{Introduction}

Weak signal detection is widely applied in radar, communication, industrial measurement and other practical engineering systems. Applying chaotic theory to weak signal detection has attracted significant attention and achieved prominent results due to its sensitivity to undetected signal and immunity to noise, in which the most proven technique is to make use of the sensitive threshold of Duffing oscillator's internal driving power to undetected signal and applying the phase patterns for signal detection or parameter estimation [Wei et al., 2009a; Wang \& He, 2003]. However, the weak signal detection by Duffing oscillator is limited as it requires corresponding phase relationship between the primary phase of the undetected signal and the primary phase of Duffing oscillator's internal driving force [Nie et al., 2007; Wang et al., 1999], which means that the existing chaotic techniques cannot detect mobile communication signal because its primary phase may change during the detection process [Wei et al., 2009b]. Currently, OFDM is the most useful method for mobile signal detection, but its calculation is complex and is limited in carrier wave, phase and hardware. Besides, we focus on weak signal detection, and OFDM is not the best method to solve it. Therefore, there is no method to realize noncoherent demodulation of DPSK mobile communication signal by Duffing oscillator, although traditional noncoherent demodulation methods to DPSK signal have been well studied.

The research of chaos-based communications has been well developed in recent years, and the most conventional technology is DCSK. The DCSK and DPSK are both noncoherent schemes and can recover the transmitted data with no channel state information. However, DCSK is much more robust to multipath fading environment, and it is far more suitable for UWB (ultra wide band) applications than DPSK, so it is widely used in security communications [Kaddoum et al., 2016; Yang \& Jiang, 2013; Xu et al., 2014]. The receiver in this study applies Duffing oscillator to traditional DPSK-modulated signal, especially for weak signal detection. The receiver preserves the capabilities of DPSK, which means that it owns better noise immunity performance and higher data rate than DCSK system.

To develop the technologies presented in [Wei et al., 2009b; Fu et al., 2011], we have formulated the mathematical equation of Duffing oscillator in this paper, and obtained the reason why a single Duffing oscillator cannot detect mobile communication signal and realizing noncoherent demodulation is because a single Duffing oscillator cannot cover the entire $360^{\circ}$ primary phase of undetected signal. Then, we present the Duffing oscillator's receiving window and research its mathematical model, as well as its movement. By matching the Duffing oscillator's receiving windows, we construct the Duffing oscillators array with the same frequency that can cover the entire $360^{\circ}$ primary phase of undetected signal. In this way, noncoherent demodulation to DPSK communication signal comes true. Besides, the traditional judgment method of the Duffing oscillator's phase pattern is dependent on human identification or algorithm, that are complex and not timely [Han \& Sun, 2011; Rigatos, 2013]. To solve this problem, the zone partition mentioned in [Fu et al., 2011] that can assess the Duffing oscillator's phase trajectory in a timely way and automatically too is used to construct the DPSK demodulator. On the base of Duffing oscillators array, zone partition and 1-bit delay technology, the DPSK signal demodulator is constructed. Combined with the weighted data fusion, the receiver may detect the signal. To improve the performance of the DPSK digital signal receiver, the Duffing oscillator optimization is proposed. It may find the optimal element in the array and its adaptive strobe technique could find the dominant element in a timely and automatic way. Finally, this new concept DPSK chaotic digital signal receiver is constructed on SystemView simulation platform and its performance is verified. Above all, the designed chaotic digital receiver (based on the Duffing oscillator optimization of Duffing oscillators array) is immune to the undetected signal primary phase and it can also reduce the BER (bit error ration) compared to the weighted data fusion. The simulation results show that the new concept receiver is better than the typical existing BPSK (2 Phase Shift Keying) receivers with significantly better SNR (signal-to-noise ratio) performance. The promotion of SNR threshold is about $0.5 \mathrm{~dB}$ than traditional chaotic method at $\mathrm{BER}=10^{-4}$ under the AWGN (additive white Gaussian noise) channel. Also, we have simulated its property in multipath Rayleigh fading channel, and the results show its stabilization.

The structure of this paper is as follows. In Sec. 2, the Duffing oscillator's mathematical model, 
receiving window and the reason for its sensitivity to the primary phase of the undetected signal are introduced. In Sec. 3, we discuss the movement method of Duffing oscillator's receiving window, and use it to construct the Duffing oscillators array. Then, we describe the details about zone partition, also we introduce the design of DPSK signal demodulator. In Sec. 5, the structure of the new concept DPSK chaotic digital signal receiver based on chaotic oscillator is discussed. Then the theory of Duffing oscillator optimization algorithm and its adaptive strobe technique are given in Sec. 6. Lastly, the performance of this new concept receiver is analyzed and conclusions are drawn on the basis of simulation results.

\section{Duffing Oscillator Mathematical Model and Its Phase Sensitive Features}

To detect mobile communication signal with chaotic oscillator, this paper utilizes the Duffing oscillator as research model owing to its equation and good chaotic features. Besides, the Duffing equation is chosen because it is a model of the classic nonlinear systems which has been extensively studied [Fu et al., 2011]. The Duffing oscillator's trajectory can be used to indicate whether the undetected signal occurs or not due to the weak signal submerged in noise being detected by Duffing oscillator via the phase trajectory transition from chaotic motion to periodic motion.

According to [Fu et al., 2011], the mathematical model of Duffing oscillator which is used to detect signal with frequency $\omega$ can be expressed as follows:

$$
\left\{\begin{aligned}
\dot{y}_{1}(t)= & \omega y_{2}(t) \\
\dot{y}_{2}(t)= & \omega\left[-k y_{2}(t)+y_{1}(t)-y_{1}^{3}(t)\right. \\
& +\gamma \cos (\omega t)+a x(t)]
\end{aligned}\right.
$$

where $y_{1}(t)$ and $y_{2}(t)$ are the state variables of the Duffing oscillator; $x(t)=s(t)+n(t)$ is the external signal injected into the Duffing oscillator, $s(t)$ is the signal to be detected, $n(t)$ is background noise, $a$ is the injection strength factor of the external signal, and $\gamma \cos (t)$ represents the Duffing oscillator's internal driving power. The value of $\gamma$ keeps the trajectory of Duffing oscillator remaining in the threshold of chaotic state which may change into large scale periodic state if undetected signal occurs. During the research, the value of $\gamma$ was decided by experiments using conditions of a specific signal.

If there is no noise source, we know from Eq. (1) that $x(t)=s(t)=\cos [(\omega+\Delta \omega) t+\varphi]$, and $\Delta \omega$ is the frequency offset of undetected signal relative to $\gamma \cos (t), \varphi$ is the primary phase of undetected signal. Now, the sum of $\gamma \cos (\omega t)$ and $a \cos [(\omega+$ $\Delta \omega) t+\varphi$ ] replaces the Duffing oscillator's internal driving power, namely:

$$
\begin{aligned}
A(t) & =\gamma \cos (\omega t)+a \cos [(\omega+\Delta \omega) t+\varphi] \\
& =\bar{\gamma}(t) \cos [\omega t+\theta(t)]
\end{aligned}
$$

where

$$
\begin{aligned}
& \theta(t)=\operatorname{arctg} \frac{a \sin (\Delta \omega t+\varphi)}{\gamma+a \cos (\Delta \omega t+\varphi)}, \\
& \bar{\gamma}(t)=\sqrt{\gamma^{2}+2 \gamma a \cos (\Delta \omega t+\varphi)+a^{2}} .
\end{aligned}
$$

If the frequency of undetected signal is equal to the frequency of Duffing oscillator's internal driving power, namely $\Delta \omega=0$, then:

$$
\begin{aligned}
\theta(t) & =\operatorname{arctg} \frac{a \sin \varphi}{\gamma+a \cos \varphi} \\
\bar{\gamma}(t) & =\sqrt{\gamma^{2}+2 \gamma a \cos \varphi+a^{2}} .
\end{aligned}
$$

Obviously, the primary phase $\varphi$ of undetected signal decides the phase trajectory of Duffing oscillator. If $\bar{\gamma}(t) \geq \gamma$, the phase trajectory of Duffing oscillator will change from the chaotic state to the large scale periodic state, which indicates the existence of undetected signal. On the other hand, if $\bar{\gamma}(t)<\gamma$, the phase trajectory of the Duffing oscillator will remain in the chaotic state, and cannot indicate the existence of the undetected signal. These corresponding phase trajectory states are shown in Fig. 1.

In conclusion, if the primary phase $\varphi$ of the undetected signal meets $\bar{\gamma}(t) \geq \gamma$, this is to say if the primary phase of undetected signal satisfies $\varphi_{1} \leq \varphi \leq \varphi_{2},\left(\varphi_{1}=-\frac{\pi}{2}-\sin ^{-1}(a / 2 \gamma), \varphi_{2}=\right.$ $\left.\frac{\pi}{2}+\sin ^{-1}(a / 2 \gamma)\right)$, the Duffing oscillator can detect the signal, otherwise, it cannot detect the signal successfully. This feature shown above is called the Duffing oscillator's receiving window and its mathematical model is as follows:

$$
\begin{aligned}
\varphi & \in\left[\varphi_{1}, \varphi_{2}\right] \\
& =\left[-\frac{\pi}{2}-\sin ^{-1}\left(\frac{a}{2 \gamma}\right), \frac{\pi}{2}+\sin ^{-1}\left(\frac{a}{2 \gamma}\right)\right] .
\end{aligned}
$$




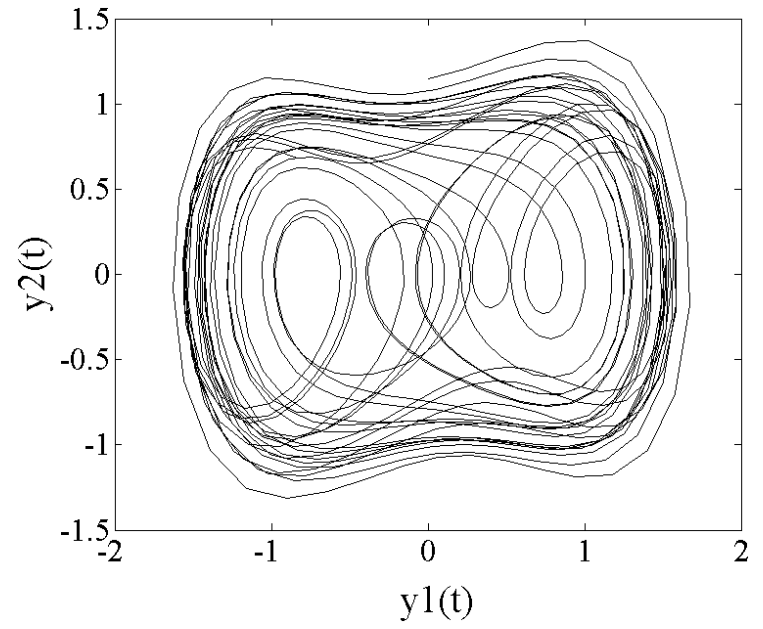

(a)

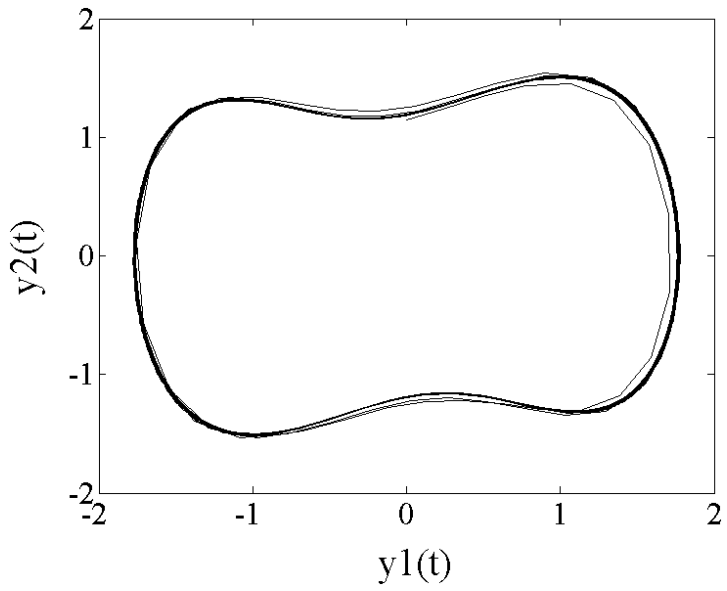

(b)

Fig. 1. The phase trajectory states of Duffing oscillator. (a) Chaotic state and (b) large scale periodic state.

If we set $a=0.1, \gamma=0.896$, the Duffing oscillator's receiving window calculated by Eq. (5) is $186.4^{\circ}\left(-93.2^{\circ}, 93.2^{\circ}\right)$. Figure 2 shows the corresponding scope of the single Duffing oscillator's receiving window whose trajectory state is sensitive to the corresponding primary phase extent of the undetected signal $(a=0.1, \gamma=0.896)$.

From Fig. 2, it is evident that a single Duffing oscillator's receiving window cannot cover the entire $360^{\circ}$ primary phase of the undetected signal. Importantly, it can only detect the signal whose primary phase falls into the Duffing oscillator's receiving window, and it is incapable of detecting the signal if its primary phase digresses from the Duffing oscillator's receiving window. This explains why a single Duffing oscillator cannot detect mobile communication signal, due to the movement of communication source resulting in the primary phase of those signals not always falling into a single fixed Duffing oscillator's receiving window.

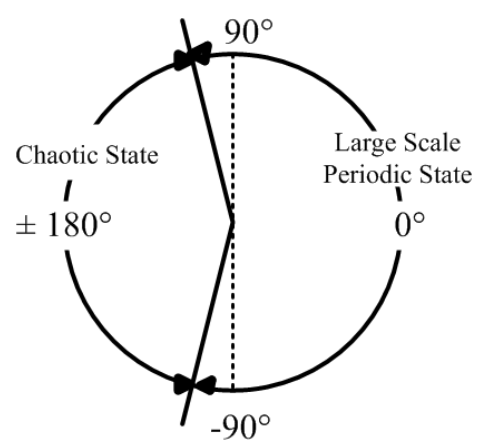

Fig. 2. The Duffing oscillator's receiving window.

\section{The Method of Using Duffing Oscillators Array to Detect Communication Signal}

\subsection{The movement of single Duffing oscillator's receiving window}

Through the theoretical analysis of single Duffing oscillator state equation and simulation results, we found that the Duffing oscillator's receiving window can be moved by shifting the primary phase of Duffing oscillator's internal driving force [Nie et al., 2007; Wang et al., 1999].

To expand the width of the Duffing oscillator's receiving window, the Duffing oscillator's mathematical model can be modified as follows:

$$
\left\{\begin{aligned}
\dot{y}_{1}(t)= & \omega y_{2}(t) \\
\dot{y}_{2}(t)= & \omega\left[-k y_{2}(t)+y_{1}(t)-y_{1}^{3}(t)\right. \\
& \left.+\gamma \cos \left(\omega t+\varphi_{0}\right)+a x(t)\right] .
\end{aligned}\right.
$$

Then, we calculate the primary phase extent of Duffing oscillator's internal driving force which is sensitive to undetected signal as Eq. (7) gives:

$$
\begin{array}{r}
\varphi \in\left[-\frac{\pi}{2}-\sin ^{-1}\left(\frac{a}{2 \gamma}\right)+\varphi_{0},\right. \\
\left.\quad \frac{\pi}{2}+\sin ^{-1}\left(\frac{a}{2 \gamma}\right)+\varphi_{0}\right] .
\end{array}
$$

According to Eq. (7), we know that the movement of the Duffing oscillator's receiving window is accomplished by adjusting $\varphi_{0}$. By matching several Duffing oscillator's receiving windows with different 
primary phases, the extension of Duffing oscillator's receiving window is realized and the signal with random primary phase $\varphi_{0}$ can be detected.

\subsection{The method of creating Duffing oscillators array}

By making use of the movement of Duffing oscillator's receiving window, it is possible to construct the Duffing oscillators array operating at the same frequency. This array can cover the entire $360^{\circ}$ primary phase fully and it is immune to the primary phase of the undetected signal, which makes it very useful for mobile communication signal detection. The structure of the Duffing oscillators array is shown as in Fig. 3.

The detailed construction method of the Duffing oscillators array is as follows:

(i) Choose $M$ Duffing oscillators with normalized frequency, we set $3 \leq M \leq 20$ based on the simulation experience and reduced the complexity of calculation.

(ii) The $M$ Duffing oscillators with normalized frequency must satisfy the following equation:

$$
\begin{gathered}
\frac{d^{2} y(t)}{d t^{2}}+\delta \frac{d y(t)}{d t}-y(t)+y^{3}(t) \\
=\gamma \cos (t)+a x(t) .
\end{gathered}
$$

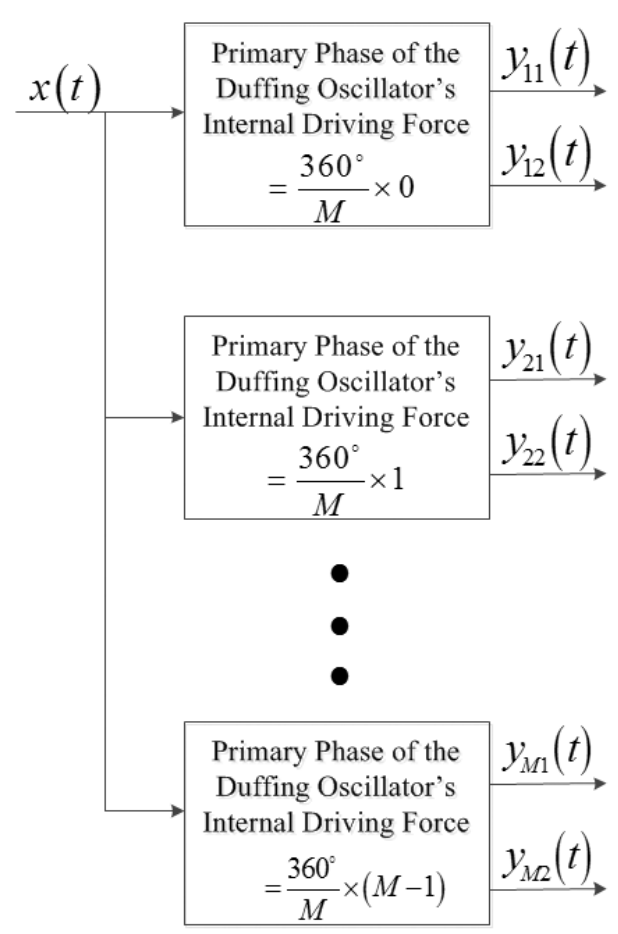

Fig. 3. Duffing oscillators array.
In Eq. (8), $x(t)=s(t)+n(t)$ is the external signal, $s(t)$ is the undetected signal with frequency $\omega, n(t)$ is the background noise, $a$ is the injection strength factor of the external signal and $\gamma \cos (t)$ is the Duffing oscillator's internal driving power.

(iii) To cover $360^{\circ}$ primary phase fully, the research adjusts the primary phase of the $M$ Duffing oscillators' internal driving power as follows:

$$
\begin{gathered}
\frac{d^{2} y(t)}{d t^{2}}+\delta \frac{d y(t)}{d t}-y(t)+y^{3}(t) \\
=\gamma \cos \left(t+\frac{360^{\circ}}{M}(i-1)\right)+a x(t) \\
(i=1, \ldots, M)
\end{gathered}
$$

(iv) Set $t=\omega \tau$, the Duffing oscillators (with a normalized frequency) can be changed into a new form that can detect a signal with frequency $\omega$.

$$
\begin{gathered}
\frac{1}{\omega^{2}} \frac{d^{2} y(\tau)}{d \tau^{2}}+\frac{\delta}{\omega} \frac{d y(\tau)}{d \tau}-y(\tau)+y^{3}(\tau) \\
=\gamma \cos \left(\omega \tau+\frac{360^{\circ}}{M}(i-1)\right)+a x(\tau) \\
(i=1, \ldots, M)
\end{gathered}
$$

(v) Substitute $\tau=t$, the research expresses Duffing oscillator's state equation as follows:

$$
\left\{\begin{array}{r}
\dot{y}_{1}(t)=\omega y_{2}(t) \\
\dot{y}_{2}(t)=\omega\left[-k y_{2}(t)+y_{1}(t)-y_{1}^{3}(t)\right. \\
\left.+\gamma \cos \left(\omega t+\frac{360^{\circ}}{M}(i-1)\right)+a x(t)\right], \\
\quad(i=1, \ldots, M) .
\end{array}\right.
$$

(vi) Send the undetected signal $x(t)$ into the $M$ Duffing state equations defined by Eq. (11), we obtained the Duffing oscillators array as shown in Fig. 3.

In Fig. 3, $y_{i 1}(t)$ and $y_{i 2}(t)$ are the outputs of Duffing oscillator $i(i=1,2, \ldots, M)$ in the array, in which the primary phase of each Duffing oscillator's internal driving power is $\varphi_{i}=\frac{360^{\circ}}{M}(i-1)$ $(i=1,2, \ldots, M)$. Each output of Duffing oscillator in the array can be calculated in a timely way by means of fourth-order Runge-Kutta method [Fu et al., 2011]. 
In the Duffing oscillators array, if mobile communication signal with an arbitrary primary phase appears, at least one Duffing oscillator's phase trajectory will change into the large scale periodic state, which can be used to detect the mobile communication signal. This feature can be used to design DPSK signal demodulator and this technique will be discussed in the next section.

To compare the performance of a single Duffing oscillator and the Duffing oscillators array with $M$ elements, Fig. 4 shows the relationship pattern of sensitivity-phase for a normalized signal. The curve

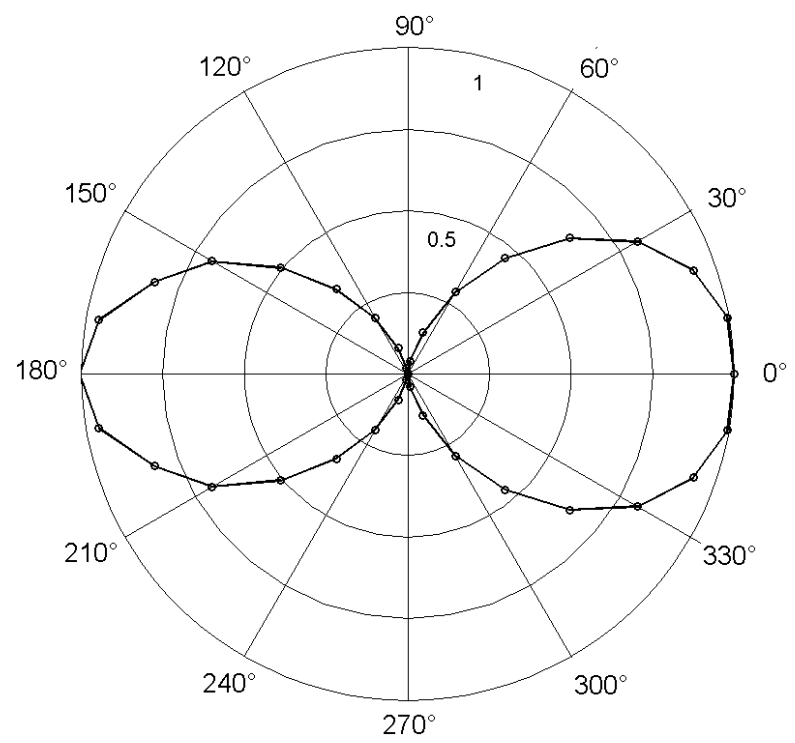

(a) $M=1$

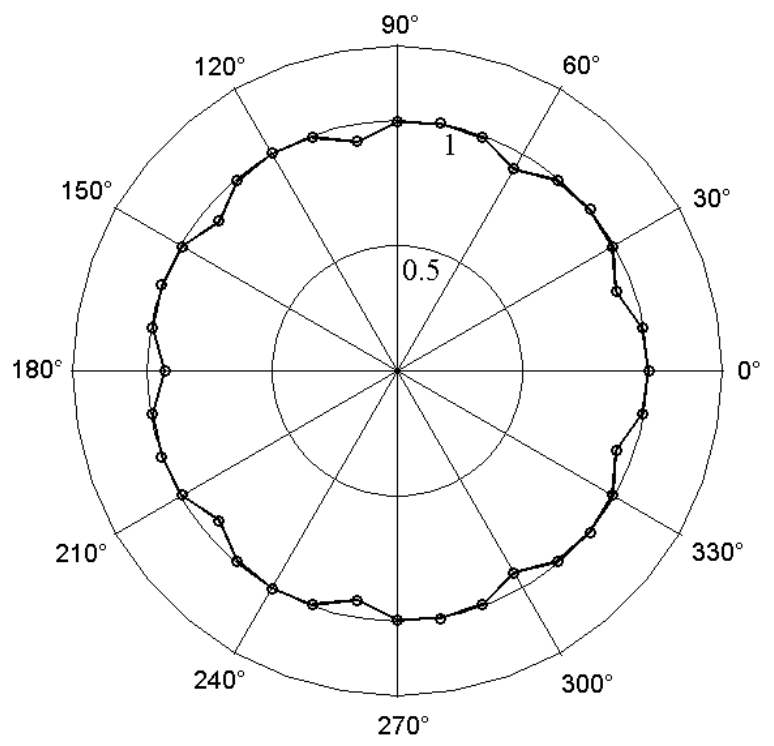

(c) $M=9$ in Fig. 4 is obtained by changing the power and primary phase of the undetected DPSK signal in the condition of BER $=10^{-3}$. From Fig. 4, comparing Figs. 4(b)-4(d), we know that the sensitivityphase is improved with the increasing of $M$, and the performance of Duffing oscillators array with $M$ elements has been improved with respect to the primary phase of the undetected signal. Moreover, the number $M$ of the array set appropriately can result in a good compromise between algorithm complexity and sensitivity-phase pattern uniformity. If $M=6$, the primary phase has to be $30^{\circ}$

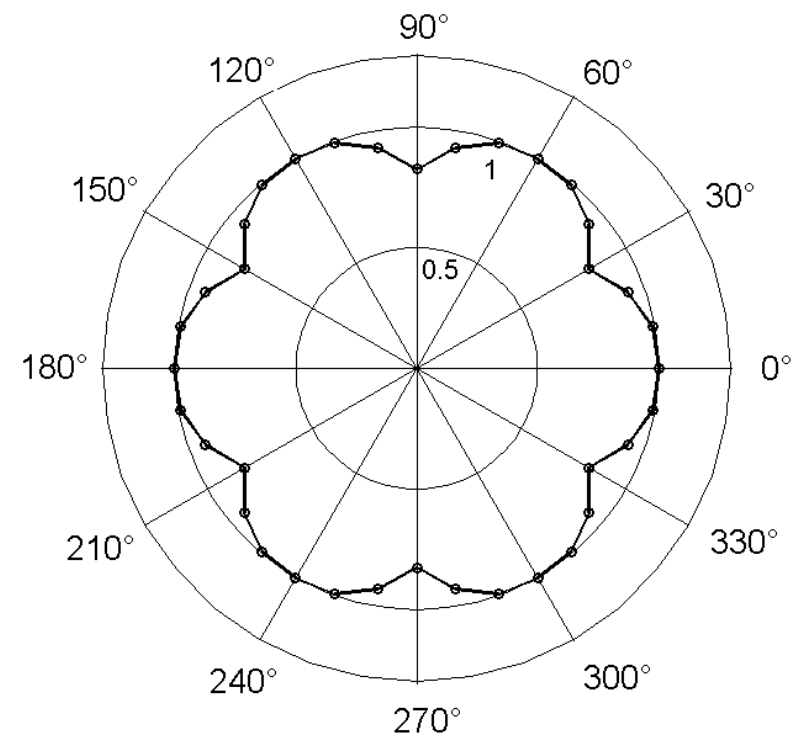

(b) $M=6$

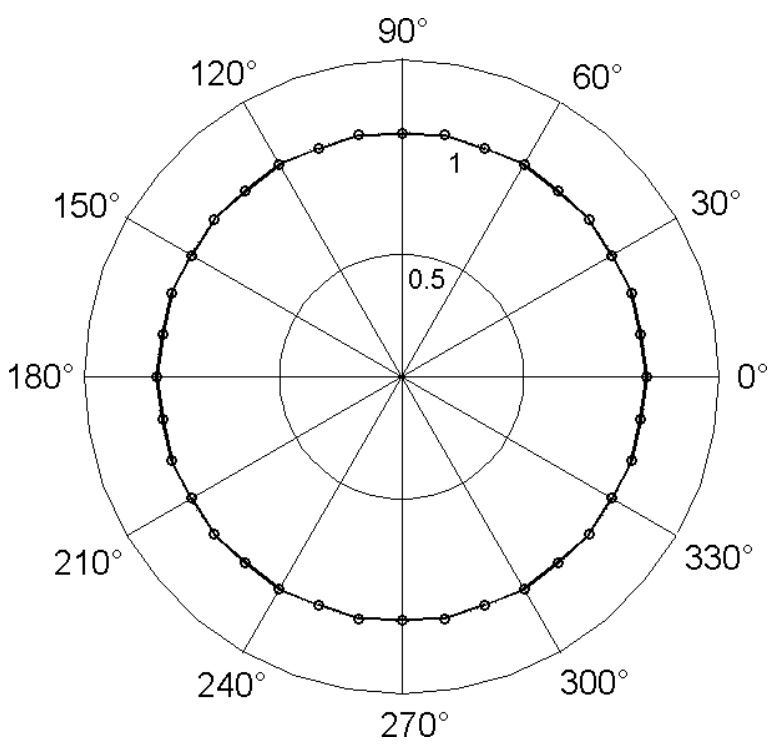

(d) $M=12$

Fig. 4. Relationship pattern of sensitivity-phase for normalized signal for DPSK communication signal demodulator. 
or else it cannot be detected well. If $M=12$, the sensitivity-phase is very good, but the algorithm is too complicated to realize. After the comparison and simulation of $M$ elements, $M=9$ is used in the receiver, for it is enough to detect any primary phase, meanwhile it is simple in algorithm.

\section{The Design of Demodulator for DPSK Communication Signal}

\subsection{The construction of the zone partition}

To detect the signal by the Duffing oscillator's phase trajectory (large scale periodic and chaotic states) in a timely and automatic way, we have to use the zone partition method proposed in [Fu et al., 2011]. The detailed theory is described as follows:

(i) Set a zone inside and never cross over the Duffing oscillator large scale periodic phase trajectory. The zone should occupy the largest area and own the simplest mathematical equation form for its boundary line.

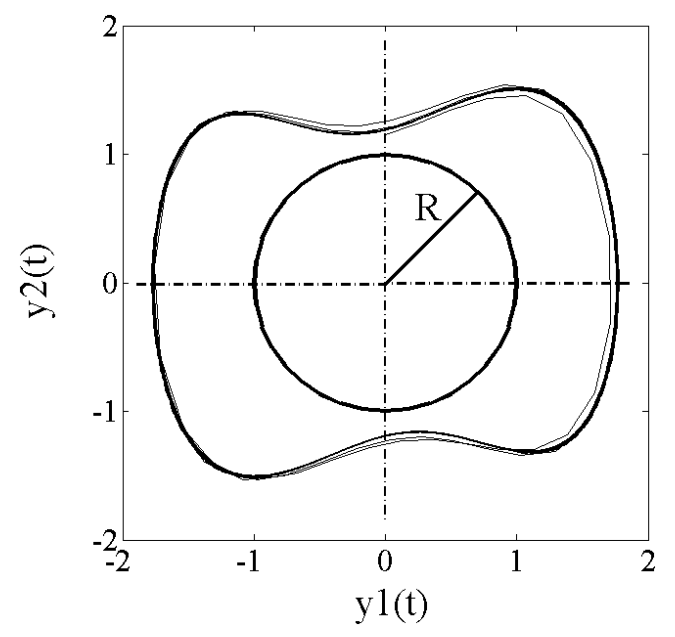

(a) (ii) " +1 " stands for the phase trajectory that does not contact and cross the boundary line of the zone partition; "-2" ("-2" may make the undetected signal's average level " -1 " after the LPF (Low Pass Filter)) stands for the phase trajectory contacting or crossing the boundary line of the zone partition.

(iii) When the output of the zone partition remains in " +1 ", this means that the undetected signal exists, otherwise, the output of the zone partition transforms between " +1 " and " -2 " frequently and this means that the undetected signal does not exist.

Then we construct the circle zone partition and the rectangle zone partition based on the theory mentioned above, with their boundary lines shown in Fig. 5.

In Fig. 5, the circle zone partition boundary line's mathematical equation is:

$$
\tilde{y}(t)= \begin{cases}+1, & \sqrt{y_{1}^{2}(t)+y_{2}^{2}(t)}>R \\ -2, & \sqrt{y_{1}^{2}(t)+y_{2}^{2}(t)} \leq R .\end{cases}
$$

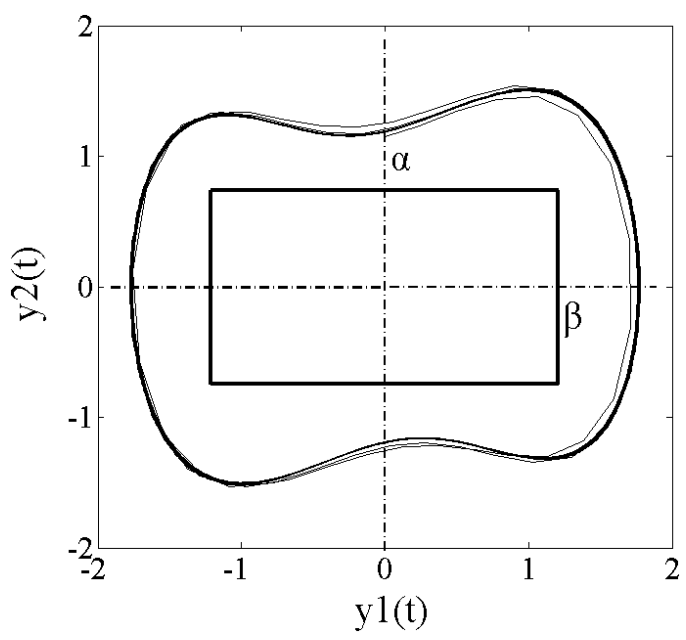

(b)

Fig. 5. Zone partition boundary line diagram. (a) Circle zone partition boundary line and (b) rectangle zone partition boundary line.

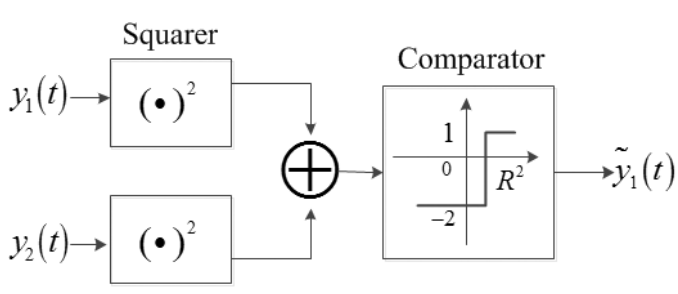

(a)

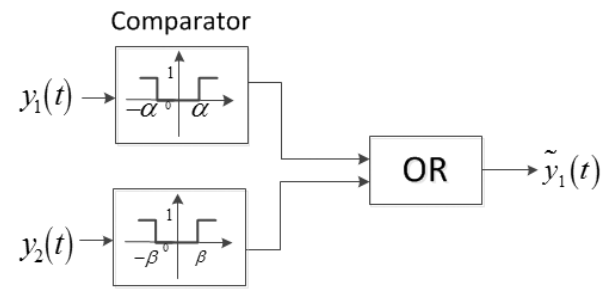

(b)

Fig. 6. Zone partition realization diagram. (a) Circle zone partition and (b) rectangle zone partition. 


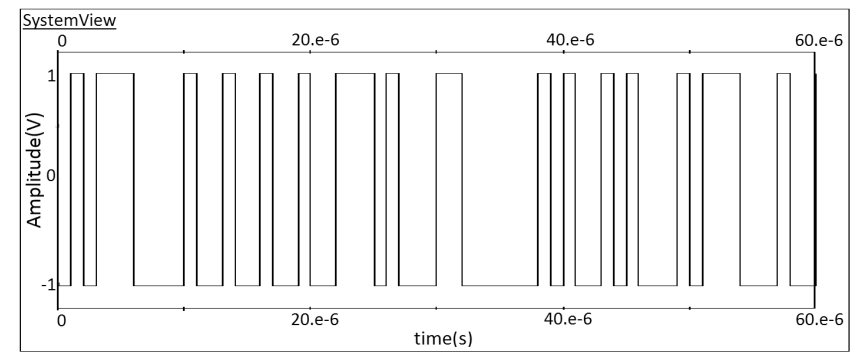

(a)

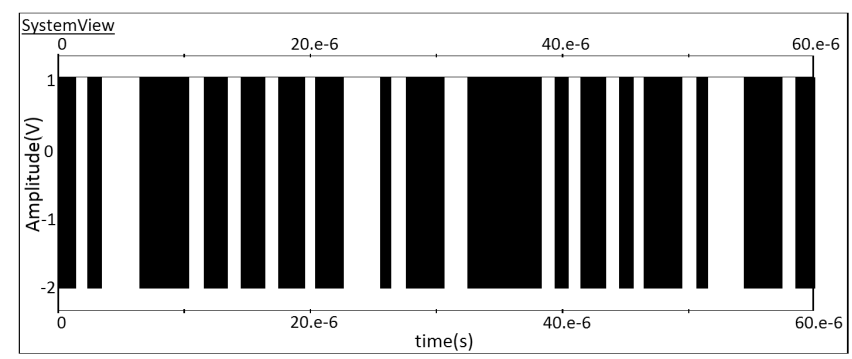

(c)

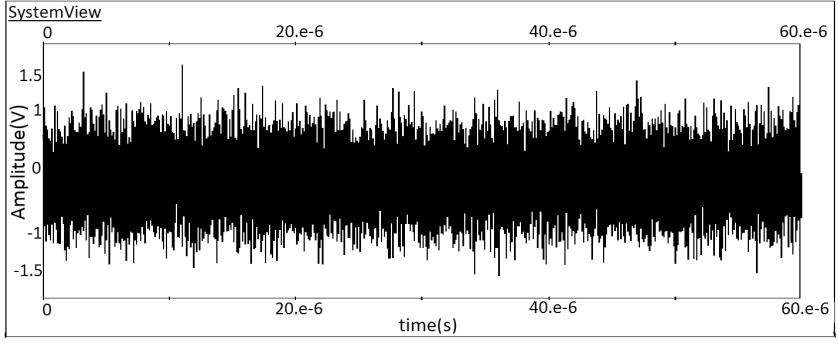

(b)

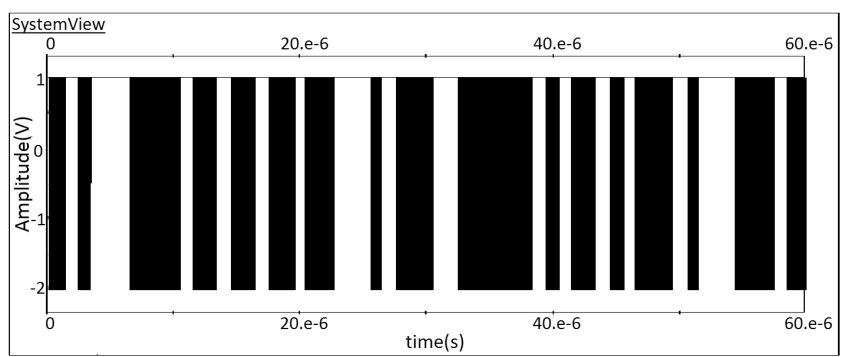

(d)

Fig. 7. Simulation results of zone partition. (a) Original signal waveform, (b) BPSK-modulated signal with noise, (c) waveform output of circle zone partition and $(\mathrm{d})$ waveform output of rectangle zone partition.

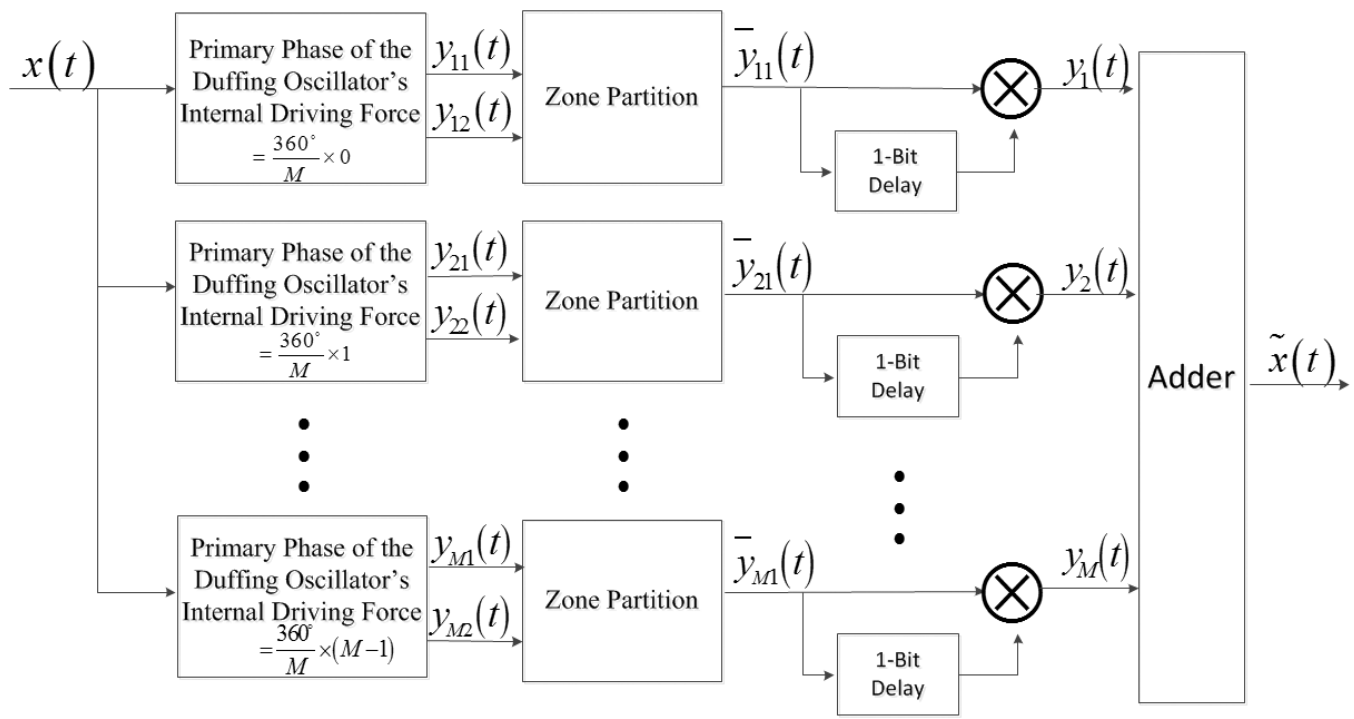

Fig. 8. Duffing oscillator DPSK communication signal demodulator.

On the other hand, the rectangle zone partition boundary line's mathematical equation is:

$$
\tilde{y}(t)= \begin{cases}+1, & \text { others } \\ -2, & \left|y_{1}\right| \leq \alpha, \quad\left|y_{2}\right| \leq \beta\end{cases}
$$

In Eq. (12), $R$ is the radius of the circle zone partition boundary line, while $\alpha$ and $\beta$ in Eq. (13) are the half dimensions of the rectangle zone partition boundary line separately. According to Eqs. (12) and (13), the practical structure of the zone partition is built as in Fig. 6 where the output of the "OR" operation is either high level " +1 " or low level " -2 ". The corresponding simulation results are shown in Fig. 7.

To identify the performance of zone partition and compare the difference of two different zone partitions, we obtain the simulation results within time length of $60 \mu \mathrm{s}$ in Fig. 7. The input 
is BPSK-modulated signal and the results are simulated under the condition of $E_{b} / N_{0}=9 \mathrm{~dB}$. Figures $7(\mathrm{c})$ and $7(\mathrm{~d})$ show the outputs of circle zone partition and rectangle zone partition separately, respectively. A comparison of (a), (c) and (d) in Fig. 7, show obviously that no matter if it is circle zone partition or rectangle zone partition the Duffing oscillator's phase pattern is identified correctly.

\subsection{The construction of the DPSK communication signal demodulator}

This section outlines how to construct a DPSK communication signal demodulator by making use of Duffing oscillators array (operating at the same frequency) and utilizing the zone partition. Firstly,

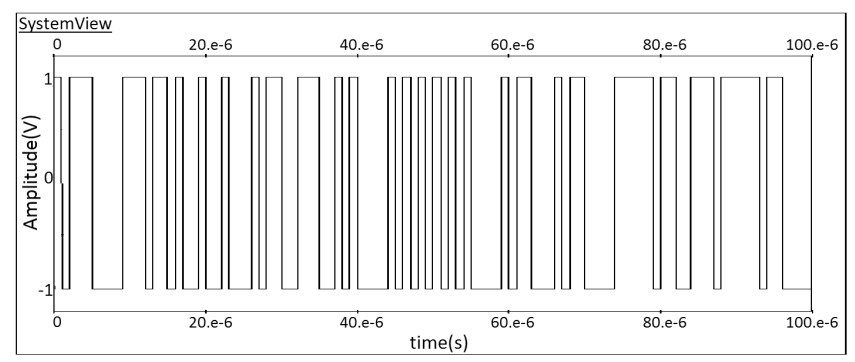

(a)

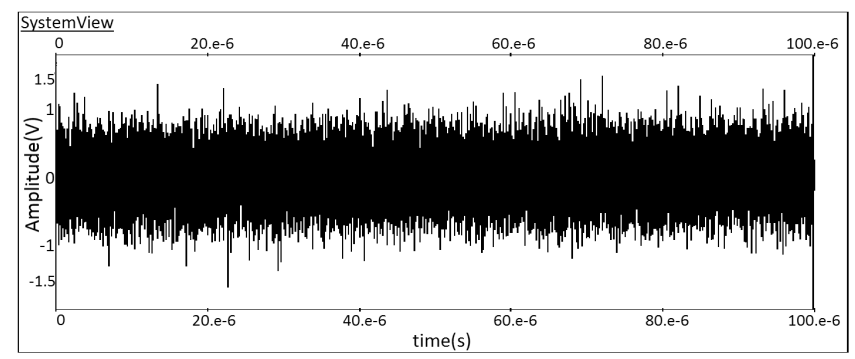

(c)

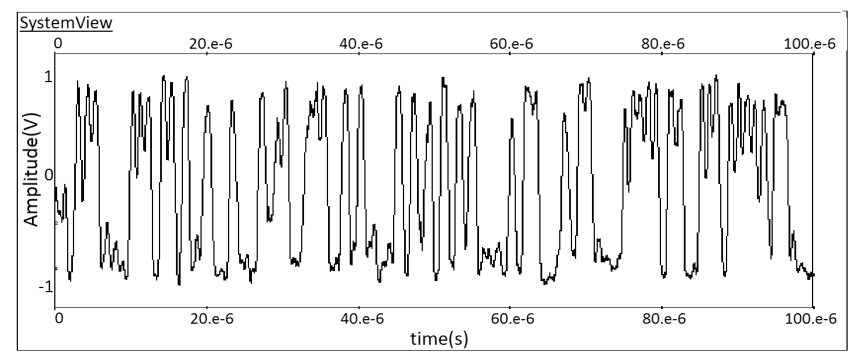

(e) assign each Duffing oscillator in the array to a zone partition which changes the two-dimensional phase trajectory containing DPSK baseband information out of Duffing oscillator into a one-dimensional $("+1$ " or "-2") DPSK baseband signal. Then use 1-bit delay to demodulate the DPSK signal and recover the original baseband information. As the primary phase of the mobile communication signal cannot always fall into a fixed Duffing oscillator's receiving window, the data fusion technique is used to extract the original communication signal information from the sum of all the zone partition outputs. According to this approach, the design of the DPSK digital signal demodulator based on Duffing oscillators array is shown in Fig. 8.

In Fig. $8, y_{i 1}(t)$ and $y_{i 2}(t)(i=1,2, \ldots, M)$ are the outputs of Duffing oscillator $i$; the zone partition

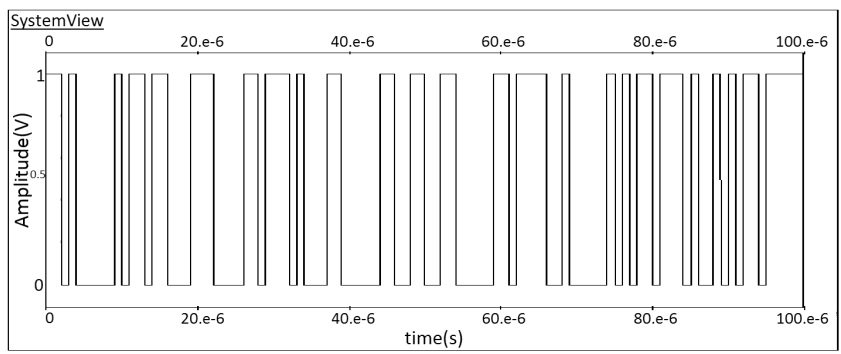

(b)

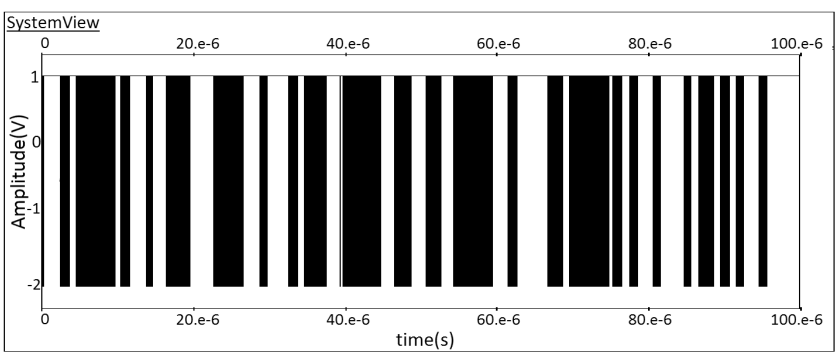

(d)

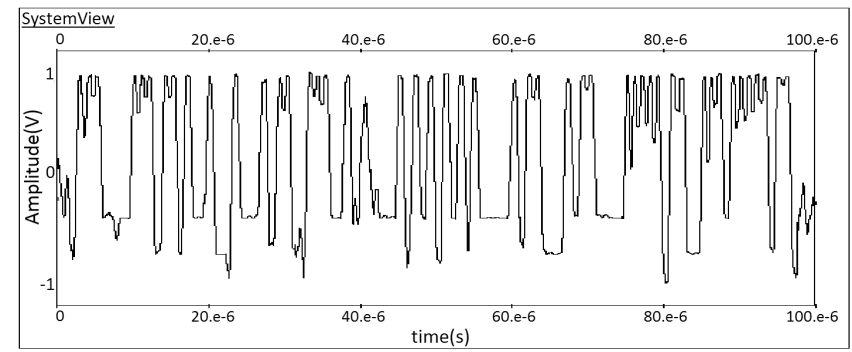

(f)

Fig. 9. Simulation results of DPSK communication signal demodulator based on Duffing oscillator. (a) Original signal waveform, (b) DPSK coding of original signal, (c) DPSK-modulated signal $s(t)$ with noise, (d) $\bar{y}_{11}(t)$ waveform output of the first zone partition, (e) $\tilde{x}(t)$ waveform output of Adder (DPSK demodulator) and (f) $\bar{y}_{o}(t)$ waveform output of optimal element. 
boundary line is assumed to be a rectangle in this paper; $\tilde{x}(t)=\sum_{i=1}^{M} \bar{y}_{i}(t)$ is the demodulated original signal and it is the output of smoothing filter in Fig. 11. Here, the sum operation of $y_{i}(t)$ is equal to the weighted data fusion, thereby allowing cooperation within the Duffing oscillators array to improve the performance of the sensitivity-phase pattern of the DPSK signal demodulator.

Figure 9 shows the simulation results of the demodulator shown in Fig. 8 by the SystemView simulation platform under the condition of $E_{b} / N_{0}=$ $9 \mathrm{~dB}$ in AWGN channel. Figure $9(\mathrm{e})$ is the waveform output from Adder, namely the output of weighted data fusion. It is evident from Fig. 9 that the noncoherent demodulation can be used to extract the original baseband signal from DPSK signal by means of the DPSK communication signal demodulator. Besides, according to Fig. 9(d) we know that the rectangle zone partition can identify Duffing oscillator's phase pattern correctly.

\section{The Design of DPSK Chaotic Digital Signal Receiver Based on Duffing Oscillator}

By making use of the DPSK demodulator given in Sec. 4, the DPSK chaotic digital signal receiver based on Duffing oscillator can be designed as in Fig. 10.

In Fig. 10, LNA is low noise amplifier, RF whose central frequency and bandwidth are $36.05 \mathrm{MHz}$ and $2 \mathrm{MHz}$ is frequency-selecting amplifier used to filter out the out-of-band interference and magnify the undetected communication signal. The zero-crossing detector is 1-bit A/D (analog to digital) converter or high speed voltage comparator. The digital signal may be high speed communication signal (in this research, the sample rate is $360.5 \mathrm{MHz}$ ) and considering the hardware realization, the $\mathrm{A} / \mathrm{D}$ converter cannot achieve the conversion speed and realize demodulation. In this research, we found that zero-crossing detector can solve the problem [Fu et al., 2011], it can be regarded as NOT gate in logical circuit and sample data with high speed, so we use it instead of $\mathrm{A} / \mathrm{D}$ converter and its output is $\pm 160 \mathrm{mV}$. The structure of the Duffing oscillator demodulator is shown in Fig. 8. An integral reset filter is used as an optimized filter to recover the original baseband information in $\tilde{x}(t)$ and bit synchronization is constructed by square law technology and replicabletriggered monostable multivibrator.

Based on the receiver mentioned in $[\mathrm{Fu}$ et al., 2011], some parameters of this new concept receiver are designed as follows. The signal into Duffing digital receiver is $x(t)=s(t)+n(t)=\bar{d}(t) A \cos \left(2 \pi f_{c} t+\right.$ $\varphi)+n(t)$, in which $d(t)$ is the original baseband data with rate $T_{c}=1 \mathrm{MHz}, \bar{d}(t)$ corresponding to DPSK coding of $d(t)$ is bipolar, $f_{c}$ is carrier frequency which is equal to $36.05 \mathrm{MHz}$, and $A$ is the amplitude of cosine carrier, equal to $900 \mathrm{mV}$. The system sampling rate is $360.5 \mathrm{MHz} . n(t)$ is zero-mean additive white Gaussian noise with power spectral density $N_{0}$. According to [Fu et al., 2011], $E_{b} / N_{0}$ (bit energy to noise power spectral density ratio) is calculated as Eq. (14) in the case that input impedance is $1 \mathrm{Ohm}$.

$$
\frac{E_{b}}{N_{0}}=10 \lg \frac{\frac{A^{2}}{2} T}{N_{0}} .
$$

The other main simulation parameters such as noise power spectral density $N_{0}$, simulation time length are obtained from [Fu et al., 2011].

Figure $9(\mathrm{e})$ is the waveform $\tilde{x}(t)$ output of Adder in Fig. 8, also it is the output of DPSK receiver. In the Duffing oscillators array, the outputs of each element is calculated by weighted data fusion. However, not all the Duffing oscillators in the Duffing oscillators array can detect the signal well at the same time, so weighted data fusion technology is not the best method for noncoherent

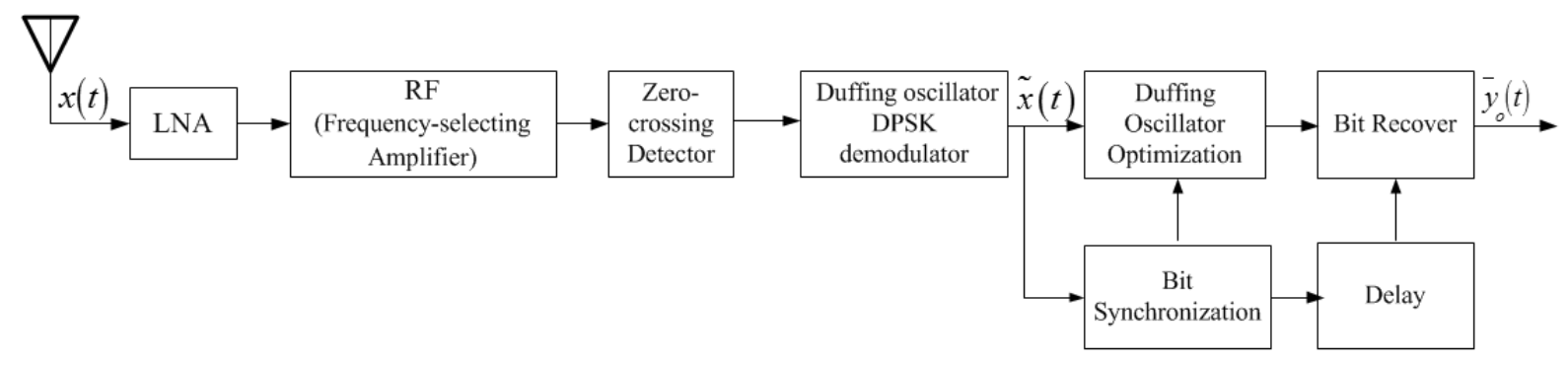

Fig. 10. DPSK receiver based on Duffing oscillator. 
demodulation. As a result, the Duffing oscillator optimization algorithm is required to elevate the receiver's performance.

\section{The Theory of Duffing Oscillator Optimization and Its Realization}

Due to the Duffing oscillator's receiving window, it is known that not all Duffing oscillators in the array may detect the signal correctly. While weighted data fusion is used to calculate the sum of outputs from all zone partitions, some oscillators in the array may reduce the overall performance of the receiver. Hence, the Duffing oscillator optimization algorithm is designed for the receiver. This algorithm aims to find out the Duffing oscillators with the best detection performance in the array, while rejecting the outputs of the oscillators not able to detect signal well. The basic concept of this optimization algorithm is given in Fig. 11.

In Fig. 11, signals $y_{1}(t), y_{2}(t), \ldots, y_{M}(t)$ are sent into adder in DPSK demodulator. The realization of the optimization algorithm is as follows:

(i) Calculate the weighted data fusion signal $\tilde{x}(t)=\sum_{i=1}^{M} \bar{y}_{i}(t)$.

(ii) Calculate the correlation integral. The integral time is set as $t=19.53125 \mu \mathrm{s}$, due to the fastest movement speed of signal source and to detect the signal timely, as well as find out the optimal Duffing oscillator in the shortest time to improve SNR threshold. Considering the integral period and detecting the mobile communication signal, the receiver has to find out the optimal Duffing oscillator in each integral period, so the paper sets $T=t=19.53125 \mu \mathrm{s}$ and finds out the maximum peak periodically in each integral period $T$.

(iii) Sum the outputs of the chaotic oscillator with maximum peak and two oscillators on its leftand-right as dominant element signal, and switch the outputs of the other chaotic oscillators. Thus the output of receiver can be expressed by Eq. (15):

$$
\bar{y}_{o}(t)=\left\{\begin{array}{c}
\bar{y}_{1}(t)+\bar{y}_{2}(t)+\bar{y}_{M}(t), \\
(i=1) \\
\bar{y}_{i-1}(t)+\bar{y}_{i}(t)+\bar{y}_{i+1}(t), \\
(i=2, \ldots, M-1) \\
\bar{y}_{M}(t)+\bar{y}_{M-1}(t)+\bar{y}_{1}(t), \\
(i=M) .
\end{array}\right.
$$

In Eq. (15), $y_{i}(t)$ is the output of the maximum peak Duffing oscillator, namely the optimal element.

(iv) The renewal of the optimal element (open or close) is done periodically, whereby the period is the integral time $T=t=19.53125 \mu \mathrm{s}$, and the output of the receiver remains unchanged between the adjacent renewal.

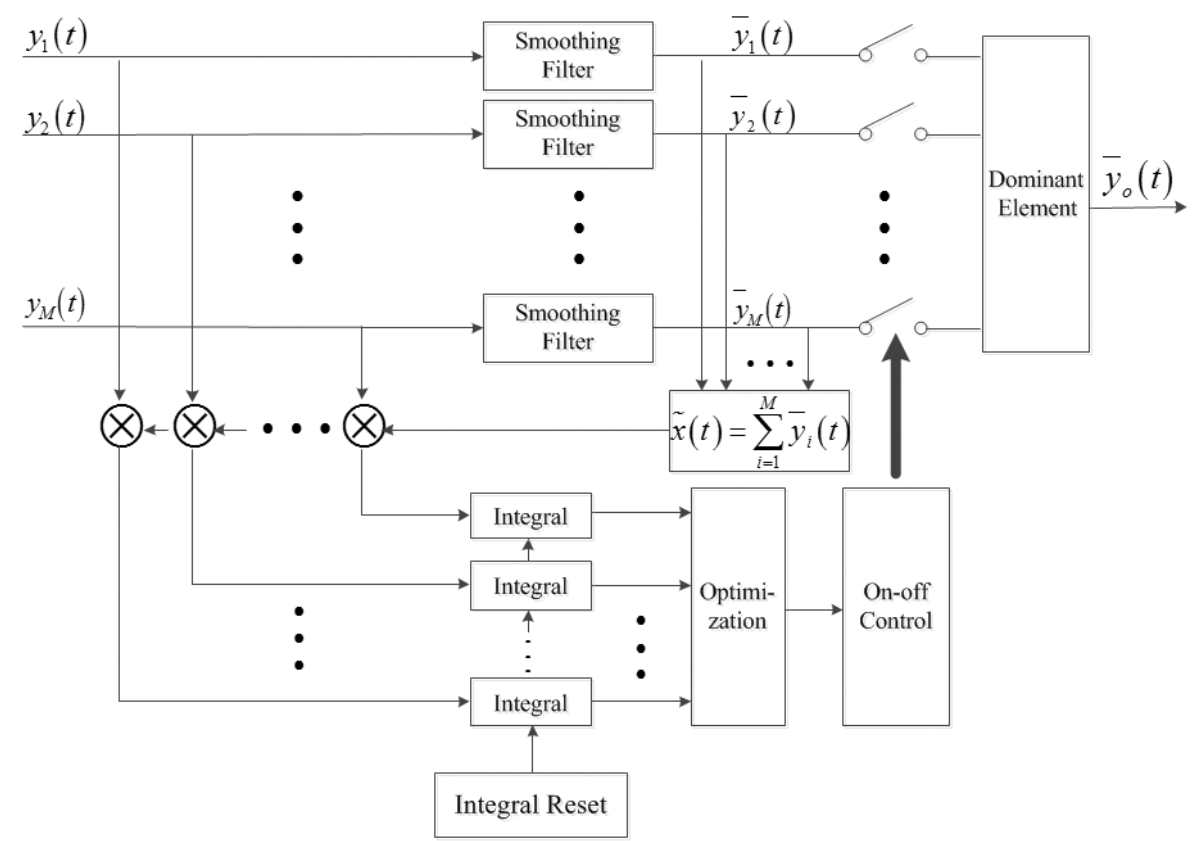

Fig. 11. Duffing oscillator optimization principle diagram. 
The first, second and third steps may find the dominant element in Duffing oscillators array, meanwhile the fourth step may realize the adaptive strobe technique. Obviously, by making use of the adaptive strobe technique of the Duffing oscillator optimization, the dominant element can be found while the outputs of some oscillators in the array will be rejected, namely the on-off control of the element which are not able to detect signals correctly is closed, which can improve the performance of SNR threshold. Figure 9(f) shows the waveform of the dominant element. From the comparison between Figs. 9(e) and 9(f), it is evident that the output of the dominant element is better than that of Adder in Fig. 8. In conclusion, the adaptive strobe technique improves the performance of the Duffing digital receiver.

\section{Simulation Results and Conclusion}

To verify the performance of the new concept DPSK chaotic digital signal receiver based on Duffing oscillators array, the simulation system is constructed on

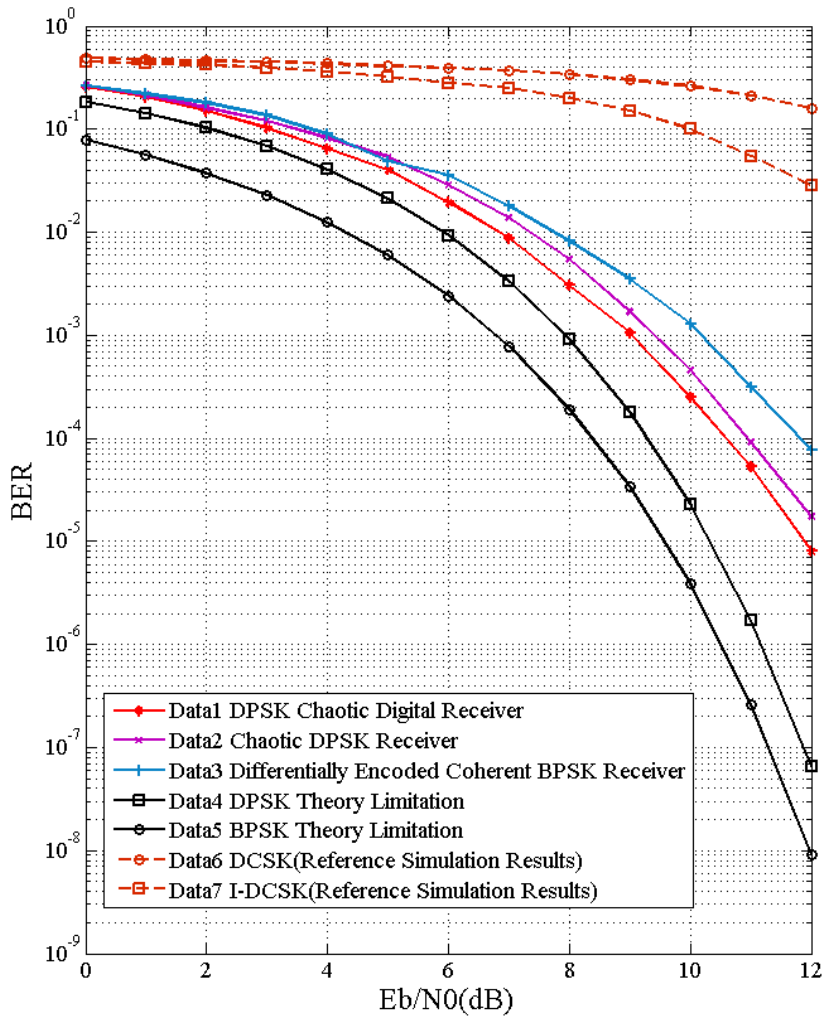

(a) the SystemView simulation platform and the corresponding BER curves are obtained in Fig. 12.

In Fig. 12(a), several BER curves are shown to compare with the new concept receiver. The detailed information of the Data1, Data2 and Data3 are given in Table 1. Data1 is the BER curve of the new concept receiver, Data2 is the BER curve of the chaotic DPSK receiver given in [Fu et al., 2011], and it is the same as the receiver without Duffing oscillator optimization of Duffing oscillators array, Data3 is the BER curve of a differentially encoded coherent BPSK receiver [Fu et al., 2011]. The detail parameters of these three receivers given in Table 1 are given in Sec. 5. Also, the DPSK receiver's theory limitation curve and the BPSK receiver's theory limitation curve are Data4 and Data5 separately. All of the BER curves are simulated under the same $E_{b} / N_{0}$ calculated by Eq. (14) as the new concept receiver in the AWGN channel except Data6, Data7. During the simulation, the corresponding receiver Data3 is obtained under the condition of fixed primary phase of carrier signal, but the Data of new concept receiver and chaotic DPSK receiver are simulated on the condition of random primary

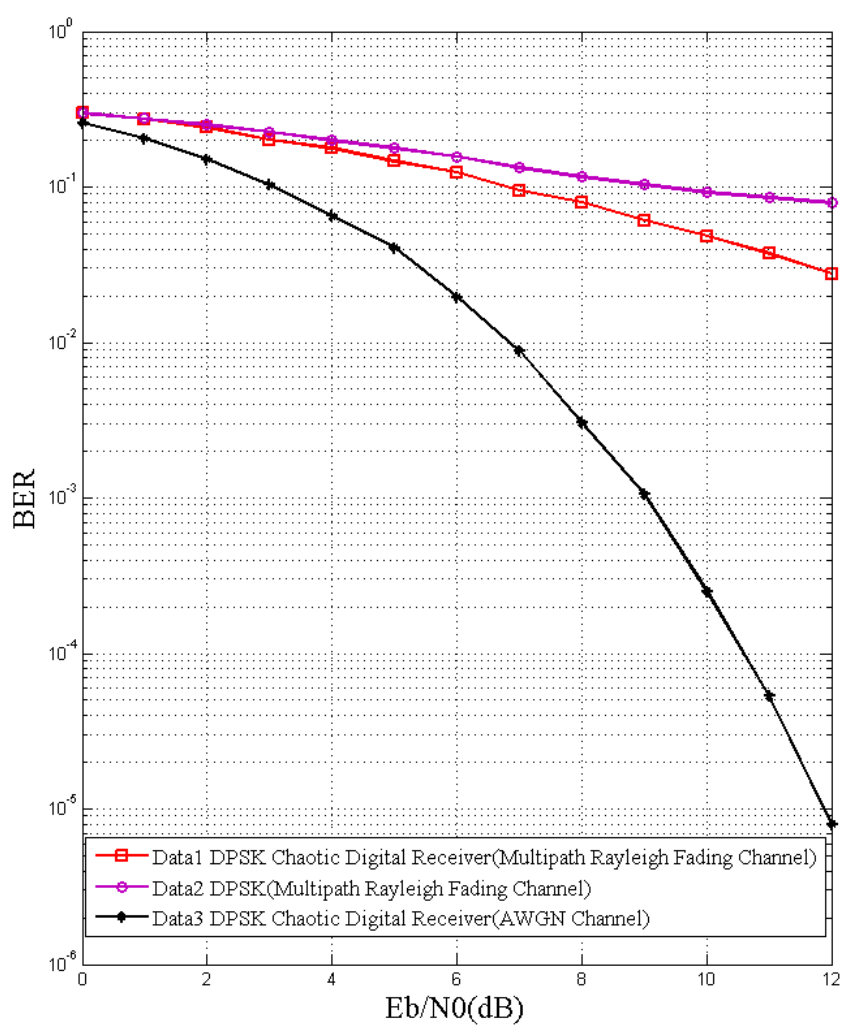

(b)

Fig. 12. Simulation results. (a) BER curves in AWGN channel and (b) BER curves in multipath Rayleigh fading channel. 
Table 1. Some information of curves in Fig. 12.

\begin{tabular}{lclcc}
\hline Curve No. & RF FILTER BW & Receiver Type & Carrier Recovery & Carrier Recovery \\
\hline Data1 & $2 \mathrm{MHz}$ & DPSK chaotic digital receiver & No & M.F. \\
Data2 & $2 \mathrm{MHz}$ & Chaotic DPSK receiver & No & M.F. \\
Data3 & $1 \mathrm{MHz}$ & Differentially encoded coherent BPSK receiver & Square-law & M.F. \\
\hline
\end{tabular}

phase for carrier signal. This means that the new concept receiver may detect the signal with any primary phase. Besides, Data6 and Data7 are simulation results of BER in the AWGN channels of DCSK and I-DCSK systems with spreading factor $\beta=200$, as researched in [Kaddoum et al., 2015]. From Fig. 12(a), we can see that the new concept receiver presented in the paper is better than the chaotic oscillator receiver given in [Fu et al., 2011] and the BPSK demodulation receiver. Notably, at $\mathrm{BER}=10^{-4}$, the promotions of SNR threshold are about $0.5 \mathrm{~dB}$ and $1.2 \mathrm{~dB}$ separately. Compared with the simulation results of chaos-based communication systems DCSK and I-DCSK presented in [Kaddoum et al., 2015], obviously the Duffing digital receiver owns better noise immunity property.

Figure 12(b) shows the simulation results in multipath Rayleigh fading channel except Data3 which shows the curves under AWGN channel. Data1 is BER performance of the new concept receiver under multipath Rayleigh fading channel with same fading parameters as Data2. Comparing Data1 and Data3, the performance of the new concept receiver in multipath Rayleigh fading channel is worse than it is in AWGN channel, this is because of the time selective fading caused by the movement of signal. Data2 is the BER curve under the multipath Rayleigh fading channel of DPSK signal where BER line under AWGN channel is Data4 in Fig. 12(a), with $f_{d} T_{c}=0.1, f_{d}$ is the Doppler shift [Zhang et al., 2010]. The BER of the new concept receiver is better than that of DPSK signal, which indicates the stable property of the new concept receiver in multipath Rayleigh fading channel.

Besides, considering the multiuser cases, the new concept digital signal receiver can be used as part of the TDMA system or OFDM system. On one hand, in the TDMA system, the signal will be modulated as BPSK/DPSK and sent at different time slots, then, the proposed receiver can be used to demodulate the signal received at the different time slots at the receiving terminal. On the other hand, in the OFDM system, the BPSK/DPSK-modulated signal is sent into OFDM system, and in that case, the new concept digital signal receiver may demodulate the signal after the carrier recovery. In short, the new concept digital signal receiver may be used in multiuser applications.

In conclusion, the design of this new concept digital signal receiver based on the Duffing oscillator optimization of Duffing oscillators array (operating at the same frequency) is completed. From the simulation results, the new concept receiver presented in this paper has better performance than typical existing receivers. In further research, we will realize the hard system of the new concept receiver, which has already been started.

\section{Acknowledgments}

The research has been funded (Grant Nos. 60772025 and 61172038 ) by the National Natural Science Foundation of China, and also through funds obtained from The Central University Basic Research Business Expenses Special Fund.

\section{References}

Fu, Y. Q., Wu, D. M., Zhang, L. \& Li, X. Y. [2011] "A circular zone partition method for identifying Duffing oscillator state transition and its application to BPSK signal demodulation," Sci. China Inform. Sci. 54, 1274-1282.

Han, J. \& Sun, H. [2011] "A study of signal detection method in power line communication based on Duffing chaotic system," Proc. IEEE Int. Symp. Microwave, Antenna, Propagation, and EMC Technologies for Wireless Communications (MAPE'11) (Beijing, China), pp. 812-815.

Kaddoum, G., Soujeri, E., Arcila, C. \& Eshteiwi, K. [2015] "I-DCSK: An improved noncoherent communication system architecture," IEEE Trans. Circuits Syst.-II: Exp. Briefs 62, 901-905.

Kaddoum, G., Soujeri, E. \& Nijsure, Y. [2016] "Design of a short reference noncoherent chaos-based communication systems," IEEE Trans. Commun. 64, 680-689.

Nie, C., Wang, H. \& Guo, W. [2007] "Detecting method of weak sine signal with initial phase," Proc. 
Int. Conf. Electronic Measurement and Instruments (ICEMI'07) (Xi'an, China), pp. 3-742-3-745.

Rigatos, G. G. [2013] "Design of chaos-based communication system with use of the derivative-free nonlinear Kalman filter," Proc. Int. Conf. Machine Learning and Applications (ICMLA'13) (Miami, FL), pp. 8-14.

Wang, G., Chen, D., Lin, J. \& Chen, X. [1999] "The application of chaotic oscillators to weak signal detection," IEEE Trans. Industr. Electron. 46, 440-444.

Wang, G. \& He, S. [2003] "A quantitative study on detection and estimation of weak signals by using chaotic Duffing oscillators," IEEE Trans. Circuits Syst.-I: Fund. Th. Appl. 50, 945-953.

Wei, C., Chen, M., Wang, C. \& Zhang, Z. [2009a] "Summary on weak signal detection methods based on chaos theory," Proc. Int. Conf. Electronic Measurement and Instruments (ICEMI'09)(Beijing, China), pp. 1-430-1-435.
Wei, C., Chen, M., Zhang, Z. \& Xu, A. [2009b] "Weak signal phase measurement method based on Duffing oscillator," Proc. Int. Conf. Electronic Measurement and Instruments (ICEMI'09) (Beijing, China), pp. 1964-1-968.

$\mathrm{Wu}, \mathrm{X}$. et al. [2001] Introduction to Chaos (Shanghai Science and Technology Press, Shanghai), pp. 35-39.

Xu, W., Wang, L. \& Chen, G. [2014] "Performance analysis of the CS-DCSK/BPSK communication system," IEEE Trans. Circuits Syst.-I: Reg. Papers 61, 26242633.

Yang, H. \& Jiang, G.-P. [2013] "Reference-modulated DCSK: A novel chaotic communication scheme," IEEE Trans. Circuits Syst.-II: Exp. Briefs 60, 232236.

Zhang, S., Tian, Y., Zhang, T. \& Zhang, J. [2010] Digital Communication Basis in Fading Channel (Harbin Engineering University Press, Harbin), pp. 101-105. 